\title{
Christian Doppler Labors - Freude und Nutzen
}

\author{
Bereicherung der Wissenschafts- und Forschungskultur
}

Online publiziert am 18. Januar 2014

Was motiviert junge Wissenschaftler und Wissenschaftlerinnen, gemeinsam mit Forschungsleitern von Partnerunternehmen einen Antrag auf Einrichtung eines Christian-Doppler-Labors zu erarbeiten und diesen Antrag hinsichtlich wissenschaftlicher Qualität sowie sich selbst auf wissenschaftliche Qualifikation und persönliches "Standing" in der Scientific Community einer strengen internationalen Evaluierung auszusetzen? - Die Antwort liegt wohl auch darin, mit einem CD-Labor das erwarten zu dürfen, was Christian Doppler mit folgenden Worten ausgedrückt hat: „Die lohnendsten Forschungen sind diejenigen, welche, indem sie den Denker erfreu'n, zugleich der Menschheit nützen." - Der Umstand, dass die Zahl der CDLabors über die fast 25-jährige Geschichte der Christian Doppler Forschungsgesellschaft ständig gestiegen ist und weiter steigt, darf als Indiz dafür gewertet werden, dass sich diese Erwartung erfüllt und auch bei den vielen CDLabors, die in der Vergangenheit an der Montanuniversität eingerichtet worden waren, erfüllt hat.

Wenn auch CD-Labors auf eine klar beschränkte Laufzeit von maximal sieben Jahren eingerichtet werden - und diese Laufzeit im Falle positiver Ergebnisse von zwei Zwischenevaluierungen auch ausschöpfen - so kann nach diesem Zeitraum keineswegs von einem "das war's" gesprochen werden. Während der Laufzeit wurden nicht nur grundlagenwissenschaftliche Erkenntnisse und Methoden zur Stärkung der Innovationskraft der Partnerunternehmen erarbeitet, und es wurden nicht nur viele Nachwuchswissenschaftler/innen zum Doktorat, bisweilen zur Habilitation geführt, sondern es wurde eine besondere Ausgangbasis für künftige Aktivitäten geschaffen:
Nicht wenige ehemalige CD-Laborleiter und -leiterinnen finden wir heute als Universitätsprofessoren und -professorinnen, einige als Mitglieder in Rektors-Teams von Universitäten, einige finden wir in hohen Positionen in Unternehmen, und, zusätzlich zum direkten Transfer der in CD-Labors erarbeiteten Erkenntnisse und Methoden in die Wirtschaft, tragen ehemalige Mitarbeiter und Mitarbeiterinnen von CD-Labors durch ihren Einstieg in F\&E-Abteilungen von Industriepartnern in besonders effektiver Weise zum Technologie-Transfer bei. Schließlich haben so manche Universitäten und Industrie-Unternehmen durch die Zusammenarbeit über CD-Labors verstärkt die Erkenntnis gewonnen: Aus einer Kooperation, in der sich beide Partner, Universität und Industrie, auf gleicher Augenhöhe treffen, in der die Erwartungen, die Bedürfnisse und die Beiträge beider Partner geachtet werden, da können beide viel Wertvolles gewinnen. - Das mag mit ein Grund dafür sein, dass das Christian-Doppler-Modell international hoch geschätzt wird und uns viele Länder, d. h. Vertreter aus diesen Ländern, um das CD-Modell beneiden. Wir aber dürfen uns freuen, es zu haben.

Franz G. Rammerstorfer Vorsitzender des wissenschaftlichen Senats, der Christian Doppler Forschungsgesellschaft, Wien, Österreich 